\title{
Confined coherence and analytic properties of Green's functions
}

\author{
K.Schönhammer \\ Institut für Theoretische Physik, Universität Göttingen, Bunsenstr. 9, Germany
}

(November 23, 2013)

\begin{abstract}
A simple model of noninteracting electrons with a separable one-body potential is used to discuss the possible pole structure of single particle Green's functions for fermions on unphysical sheets in the complex frequency plane as a function of the system parameters. The poles in the exact Green's function can cross the imaginary axis, in contrast to recent claims that such a behaviour is unphysical. As the Green's function of the model has the same functional form as an approximate Green's function for coupled Luttinger liquids no definite conclusions concerning the concept of "confined coherence", can be drawn from the locations of the poles of this Green's function.
\end{abstract}

Recently the concept of "confined coherence" was introduced in the theoretical description of quasi-one dimensional conductorst. Typically one considers two identical chains of strongly interacting electrons which are prepared in their isolated chain ground states but with one chain having $\delta N$ more electrons than the other. A transverse hopping $t_{\perp}$ is switched on and the behaviour of $\langle\delta N(t)>$ is studied. It is conjectured that the disappearance of interference oscillations in $\langle\delta N(t)\rangle$ represents a generic loss of coherence, which implies that e.g. the single particle Green's function should not exhibit a pole on the real axis which disperses with the perpen dicular momentum. In this context Clarke and Strong examined the behaviour of the approximate Green's finction for coupled Luttinger liquids proposed by Went. In their discussion these authors argue that if a pole crosses the imaginary axis on an unphysical sheet of the complex frequency plane this signals a transition between qualitatively different phases of matter. In this short note we present simple model, where the exact Green's function in fact shows such a behaviour without any qualitative change of the low energy spectral properties at the crossing. This shows that it is diffcult to draw definite conclusions from such a scenario.

We study the model of a half filled symmetric band of noninteracting spinless fermions perturbed by a separable one body potential

$$
H=\sum_{\mathbf{k}} \epsilon_{\mathbf{k}} c_{\mathbf{k}}^{\dagger} c_{\mathbf{k}}+V_{0} a^{\dagger} a
$$

where the fermion operator $a$ is given by

$$
a=\sum_{\mathbf{k}} \alpha_{\mathbf{k}} c_{\mathbf{k}}
$$

and the sum of $\left|\alpha_{\mathbf{k}}\right|^{2}$ is assumed to be unity. Using the equation of motion method 3 the calculation of the single particle Green's functions is straightforward. For $\operatorname{Im} z>$ $0(\operatorname{Im} z<0)$ the retarded (advanced) Green's function $G_{a a}(z) \equiv\left\langle\left\langle a ; a^{\dagger}\right\rangle\right\rangle_{z}$ is given by

$$
G_{a a}(z)=\frac{G_{a a}^{(0)}(z)}{1-V_{0} G_{a a}^{(0)}(z)},
$$

with

$$
G_{a a}^{(0)}(z)=\int_{-B}^{B} \frac{\rho_{a}^{(0)}(\epsilon)}{z-\epsilon} d \epsilon
$$

where the normalized spectral function $\rho_{a}^{(0)}(\epsilon)$

$$
\rho_{a}^{(0)}(\epsilon)=\sum_{\mathbf{k}}\left|\alpha_{\mathbf{k}}\right|^{2} \delta\left(\epsilon-\epsilon_{\mathbf{k}}\right)
$$

can be chosen arbitrarily. We first assume this spectral weight to be largest in the middle of band

$$
\rho_{a}^{(0)}(\epsilon)=\frac{\gamma}{B^{2 \gamma}} \Theta\left(B^{2}-\epsilon^{2}\right)|\epsilon|^{2 \gamma-1},
$$

where $\Theta(\cdot)$ is the step function and $B$ is half the bandwidth. We assume $\gamma$ to be in the range $0<2 \gamma<1$. As $\rho_{a}^{(0)}(\epsilon)$ is symmetric, the spectral representation in Eq.(6) can be written as

$$
G_{a a}^{(0)}(z)=2 z \frac{\gamma}{B^{2 \gamma}} \int_{0}^{B} \frac{\epsilon^{2 \gamma-1}}{z^{2}-\epsilon^{2}} d \epsilon=z \frac{\gamma}{B^{2 \gamma}} \int_{0}^{B^{2}} \frac{u^{\gamma-1}}{z^{2}-u} d u .
$$

In the following we discuss retarded Green's functions which are analytic in the upper half plane. On the positive imaginary axis $G_{a a}^{(0)}$ is given by

$$
G_{a a}^{(0)}(i y)=-i y \frac{\gamma}{B^{2 \gamma}} \int_{0}^{B^{2}} \frac{u^{\gamma-1}}{y^{2}+u} d u
$$

Assuming $0<y \ll B$ and using our assumption about $\gamma$, the upper limit of the integration can be replaced by $\infty$. Then $G_{a a}^{(0)}(i y)$ can be calculated analytically

$$
G_{a a}^{(0)}(i y)=\frac{1}{i y}\left(\frac{-(i y)^{2}}{B^{2}}\right)^{\gamma} \frac{\gamma \pi}{\sin \gamma \pi} .
$$

For arbitrary $\operatorname{Im} z>0$ we just have to replace $i y$ by $z$

$$
G_{a a}^{(0)}(z)=\frac{1}{z}\left(\frac{-z^{2}}{B^{2}}\right)^{\gamma} \frac{\gamma \pi}{\sin \gamma \pi} .
$$


For $\gamma$ irrational this function has infinitely many Riemann sheets and one has to identify the "physical sheet" defined by Eq. (4). If we write $z=|z| e^{i \phi}$ we have

$$
\left(-z^{2}\right)^{\gamma}=|z|^{2 \gamma} e^{i\left[\frac{\pi}{2}(1+2 n)+\phi\right] 2 \gamma}
$$

where $n \in \mathbf{Z}$ has to be chosen properly. We use the fact that the phase of $G_{a a}^{(0)}$ has to be $-\pi / 2$ on the positive imaginary axis as seen from Eq. (8). This requires $n=$ -1 , i.e.

$$
G_{a a}^{(0)}(|z|, \phi)=\frac{1}{B}\left(\frac{|z|}{B}\right)^{2 \gamma-1} \frac{\gamma \pi}{\sin \gamma \pi} e^{i \Phi_{R}(\phi)},
$$

with $\Phi_{R}(\phi)=(\phi-\pi / 2) 2 \gamma-\phi$. It is easy to check that $-\operatorname{Im} G_{a a}^{0} / \pi$ is different from zero for $0<\phi<\pi$ and yields the spectral function in Eq. (6) when $z$ approaches the real axis from above. If $\phi$ in Eq. (12) is allowed to vary from minus to plus infinity one obtains the function on the complete Riemann surface.

The fact that $-\operatorname{Im} G_{a a}^{0} / \pi$ is different from zero for $0<\phi<\pi$ implies that the full Green's function $G_{a a}(z)$ in Eq. (3) is analytic for $0<\phi<\pi$ as every exact retarded Green's function. It has poles on the unphysical sheets corresponding to $\phi$ values outside this range. In the following we discuss a repulsive interaction $V_{0}>0$. Then a necessary condition for $G_{a a}(z)$ to have a pole is $\Phi_{R}\left(\phi_{(m)}\right)=2 \pi m$, where $m$ is integer, i.e. $\phi_{(m)}=-(\pi \gamma+2 \pi m) /(1-2 \gamma)$. The absolute value of $z$ at the poles is independent of $m$ and given by

$$
\left|z_{(m)}\right|=B\left(\frac{V_{0}}{B} \frac{\gamma \pi}{\sin \gamma \pi}\right)^{\frac{1}{1-2 \gamma}}
$$

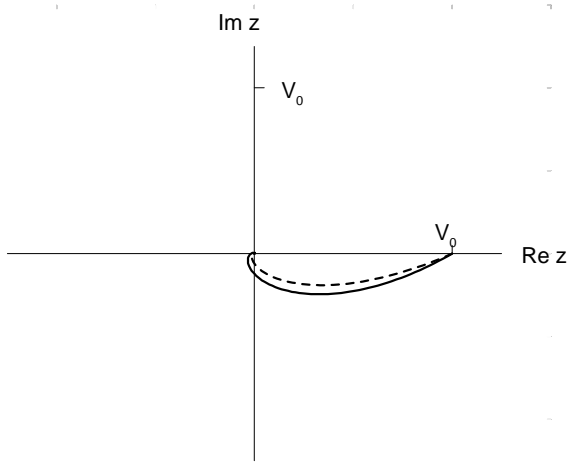

FIG. 1. Projection of the $m=0$ pole location on the complex frequency plane as a function of $\gamma$ for different values of $V_{0} / B$ (dottet line: $V_{0} / B=0.1$, dashed line: $V_{0} / B=0.05$ ). For infinitesimal $\gamma$ the pole is at $V_{0}-i 0$, and for $\gamma \rightarrow 1 / 2$ approaches the origin circling it on the different sheets of the Riemann surface. which reduces to $V_{0}$ for $\gamma \rightarrow 0$. For infinitesimal $\gamma$ the pole for $m=0$ is in the forth quadrant just below the real axis. As shown in Fig. 1 for two different values of $V_{0} / B$ it moves away from the real axis when $\gamma$ is increased, crosses the negative imaginary axis for $\gamma=1 / 4$ and circles the origin of the complex $z$-plane infinitely many times when approaching $\gamma=1 / 2$. The latter fact cannot really be seen in the figure as $\left|z_{(0)}\right|$ goes to zero very quickly near $\gamma=1 / 2$.

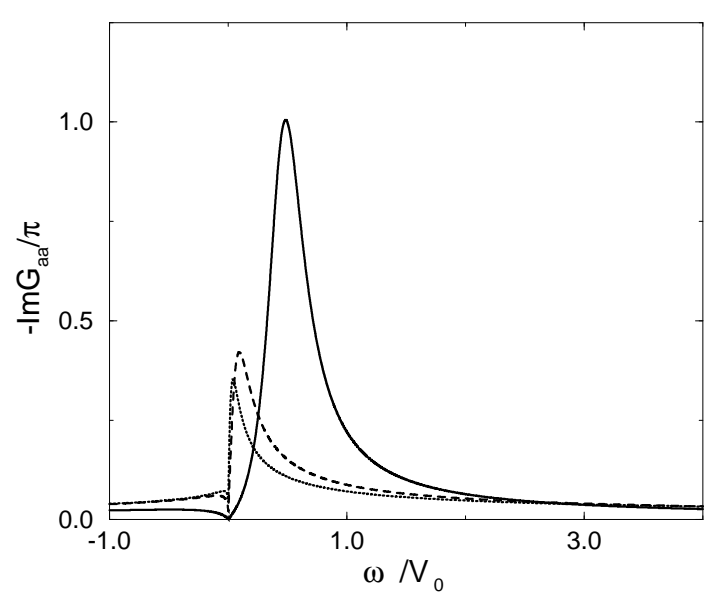

FIG. 2. Spectral function of the exact Green's function $G_{a a}$ as a function of $\omega / V_{0}$ for $V_{0} / B=0.05$ and different values of $\gamma$ (full line: $\gamma=0.1$, dashed line: $\gamma=0.23$, dotted line: $\gamma=0.27)$. No qualitative change occurs at $\gamma=1 / 4$, where the pole on the unphysical sheet crosses the negative imaginary axis.

The spectral function $-\operatorname{Im} G_{a a}(\omega+i 0) / \pi$ is shown in Fig.2 for different values of $\gamma$ with $V_{0} / B$ fixed. Changing this ratio only changes the scales of the functions. For $\gamma \ll 1$ a well defined peak occurs with the position and width determined by the position of the pole on the unphysical sheet. With increasing $\gamma$ the weight for negative $\omega$ and for $\omega \gg V_{0}$ increases. No qualitative change occurs when the pole on the unphysical sheet crosses the negative imaginary axis for $\gamma=1 / 4$. To show this we present results for $\gamma=0.23$ and $\gamma=0.27$. Also for $1 / 4<\gamma<1 / 2$ the larger weight remains in the positive frequency range. The pole on the unphysical sheet determines the spectral function in an essential way only if it is close to the physical sheet and well separated from all other non-analyticities like the branch point in the origin.

As a second example we discuss an asymmetric spectral density

$$
\rho_{a}^{(0)}(\epsilon)=\frac{\gamma}{\left(B^{2}-\epsilon_{0}^{2}\right)^{\gamma}}\left(\epsilon^{2}-\epsilon_{0}^{2}\right)^{\gamma} /\left|\epsilon-\epsilon_{0}\right|,
$$

for $\epsilon_{0}^{2}<\epsilon^{2}<B^{2}$ and zero elsewhere, where $0<\epsilon_{0} \ll B$. It has a small gap from $-\epsilon_{0}$ to $\epsilon_{0}$ in the center of the band and a power law divergence when $\epsilon$ approaches $\epsilon_{0}$ 
from above. If $\epsilon_{0}^{2}$ is neglected compared to $B^{2}$ the corresponding Green's function $G_{a a}^{(0)}$ for $|z| \ll B$ is obtained by replacing $1 / z$ in Eq. (10) by $1 /\left(z-\epsilon_{0}\right)$ and $z^{2}$ in the parenthesis by $z^{2}-\epsilon_{0}^{2}$. The correct phase can be determined similarly to the $\epsilon_{0}=0$ case. Figure 3 shows corresponding spectral functions $-\operatorname{Im} G_{a a}(\omega+i 0) / \pi$ for a repulsive one-body potential for two different values of $\gamma$. For the smaller value $\gamma=0.3$ the real part of the pole on the unphysical sheet is larger than $\epsilon_{0}$ while for $\gamma=0.36$ it is smaller than $\epsilon_{0}$. This leads to no qualitative difference in the spectral functions.

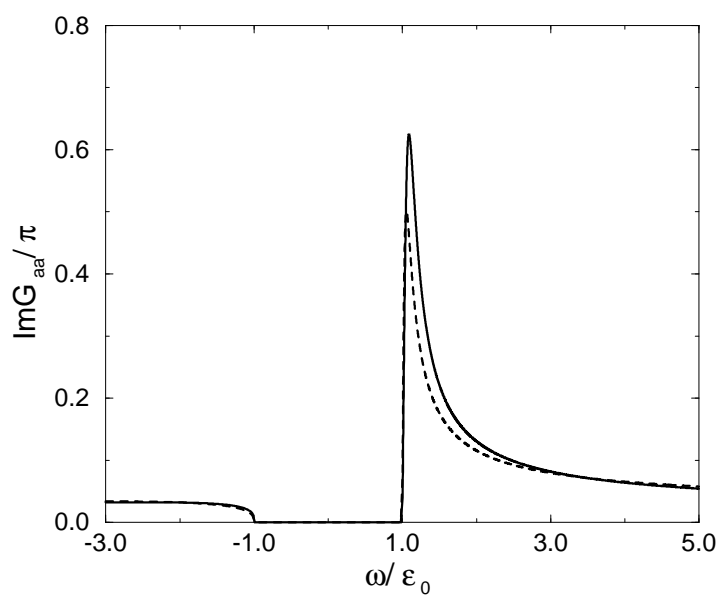

FIG. 3. Spectral function of the exact Green's function $G_{a a}$ for the asymmetric spectral density in Eq.(14) and a repulsive interaction. The parameters chosen are $V_{0} / \epsilon_{0}=0.5$ and $V_{0} / B=0.05$. The full (dotted) curve corresponds to $\gamma=0.3$ $(\gamma=0.36)$.

For attractive one-body potentials the spectral functions $-\operatorname{Im} G_{a a} / \pi$ have an additional delta peak in the gap which results from a pole on the real axis on the physical sheet. Its weight vanishes for $\left|V_{0}\right| / \epsilon_{0} \rightarrow 0$ proportional to $\left(\left|V_{0}\right| / \epsilon_{0}\right)^{\gamma /(1-\gamma)}$.

The low energy form Eq. (10) of the Green's function $G_{a a}^{(0)}$ of our simple model of noninteracting electrons has the same form as the low energy Green's fupction $g\left(k_{F}, z\right)$ of interacting fermions in one dimensione, when $2 \gamma$ is identified with the anomalous dimension $\alpha$. If one puts $\epsilon_{0}=v_{c}\left(k_{\|}-k_{F}\right)$ in Eq. (14) this corresponds to the case $k_{\|} \neq k_{F}$ for spinless fermions 2 . If a perpendicular hopping $t_{\perp}$ between a lattice of chains is treated in leading order perturbation theory in $t_{\perp}$ the resulting approximate Green's function of the coupled system for $\mathbf{k}=\left(k_{F}, \mathbf{k}_{\perp}\right)$ has the form of $G_{a a}$ in Eq. (3) when $V_{0}$ is replaced by $t_{\perp}\left(\mathbf{k}_{\perp}\right)$. This approximate Green's function 2 for coupled Luttinger liquids implies Fermi liquid behaviour for $\alpha<1$ and "confinement" for $\alpha>1$, in agreement with renormalization group arguments. It should be pointed out that for $\alpha<1$ this Green's functions shows various deviations from usual Fermi liquid behaviour.

Clarke and Strongl present evidence that the interfer- ence oscillations $\langle\delta N(t)>$ disappear for a value of the anomalous dimension $\alpha=1 / 2$ (i.e. $\gamma=1 / 4$ ) or smaller. They expect to see this "transition" also in the behaviour of the one-particle Green's function. As Wen's Green's function for $k_{\|}=k_{F}$ shows the crossing of the negative imaginary axis of the pole on the unphysical sheet at $\gamma=1 / 4$ they take this as evidence for their conjecture. Our simple model shows that there is no general physical principle to forbid such a crossing and therefore at least in Wen's approximation the single particle Green's function shows no sign of a sudden loss of coherence if it in fact occurs for $\alpha<1$.

The author would like to thank P.Kopietz and V.Meden for discussions and useful suggestions on the manuscript.

${ }^{1}$ D.C.Clarke and S.P.Strong, J.Phys.:Condens. Matter 9, 3853-3860 (1997) and Adv. in Phys., in press.

${ }^{2}$ X.G.Wen, Phys. Rev.B 42, 6623 (1990).

${ }^{3}$ D.Z. Zubarev, Sov. Phys. Usp. 3, 320 (1960). 